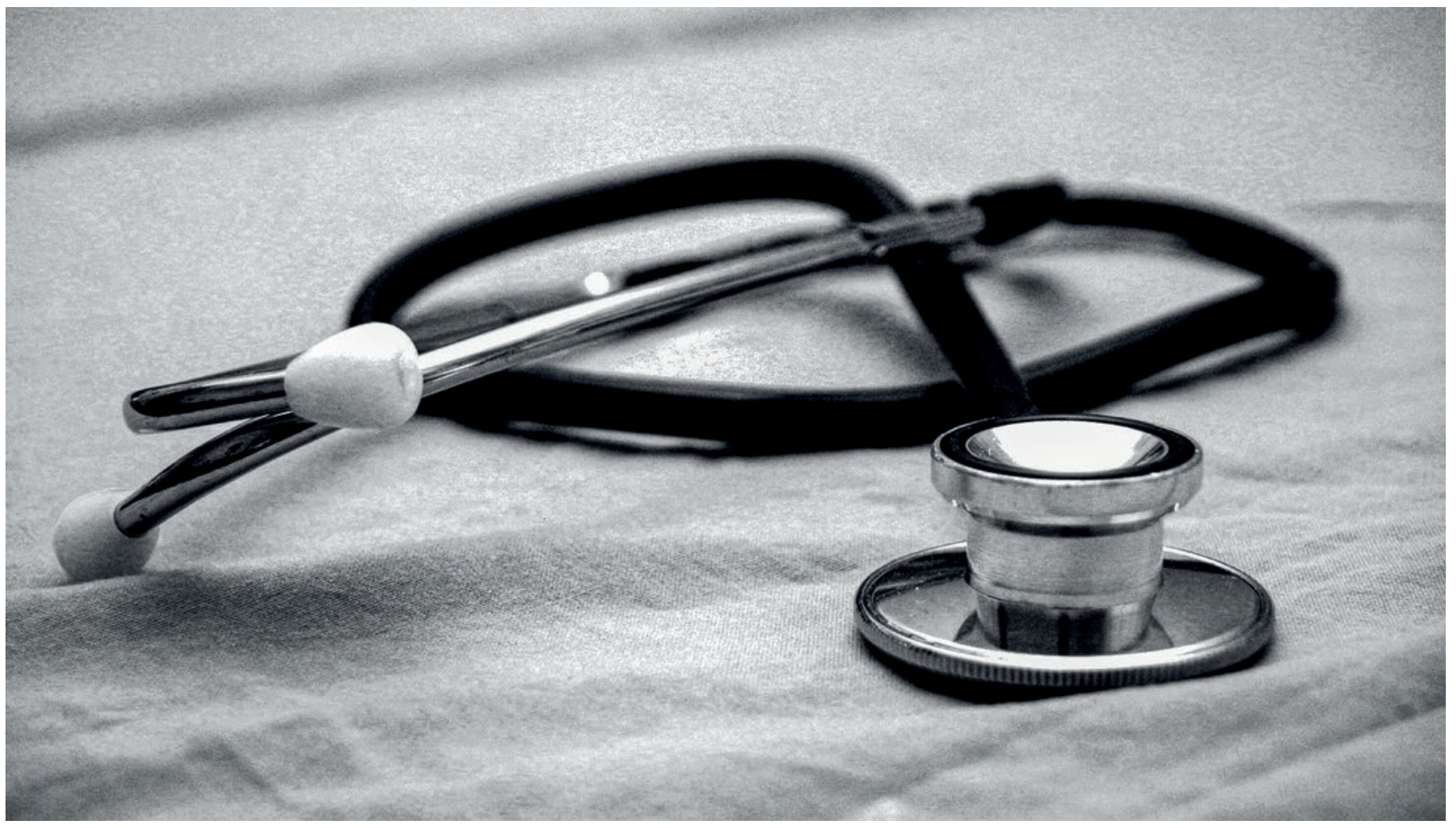

\title{
100 Jahre Medical Humanities
}

\section{Leander Diener ${ }^{\mathrm{a}}$, Flurin Condrau ${ }^{\mathrm{b}}$}

a Dr. phil., Universität Zürich, Institut für Biomedizinische Ethik und Medizingeschichte;

${ }^{b}$ Prof. Dr. phil., Universität Zürich, Institut für Biomedizinische Ethik und Medizingeschichte

Am 4. Dezember 2021 feiert die Schweizerische Gesellschaft für Geschichte der Medizin und der Naturwissenschaften (SGGMN) ihren einhundertsten Geburtstag. Es mag überraschen, dass insbesondere die Medizingeschichte vor hundert Jahren eine «angewandte Disziplin» war, ganz ähnlich den «Medical Humanities» von heute. Medizingeschichte erfüllte immer eine bestimmte Funktion in der ärztlichen Ausbildung und Praxis. Es lohnt sich, an diese weiterhin aktuelle Funktion zu erinnern.

\section{Ganzheitliche Medizin}

In ihrem vielbeachteten SÄZ-Editorial zur Frage, ob sich die FMH in umweltpolitischen Themen engagieren soll, spricht die FMH-Präsidentin Yvonne Gilli an, «dass Gesundheitsaspekte in allen Politikbereichen eine Rolle spielen» [1]. Diese Omnipräsenz von Gesundheit und Krankheit - in Umweltfragen, aber auch in wirtschafts-, sozial- und bildungspolitischen Fragen impliziere allerdings nicht, dass «medizinische Evidenz» mit spezifischem Sachwissen in der jeweiligen politischen Frage zu verwechseln sei. Oder in anderen Worten: Das Wissen um die verschiedenen Dimensionen und Faktoren von Gesundheit und Krankheit erlaubt nicht per se eine eindeutige politische Position, weil diese Expertise (wie übrigens im Allgemeinen das meiste Sachwissen auch) verschieden interpretiert werden kann. Darum sei es auch nicht die Aufgabe der FMH, sich umweltpolitisch zu engagieren. Ohne die Frage nach der Gültigkeit dieser Schlussfolgerung aufzuwerfen, fällt das umfassende, ganzheitliche Verständnis von Medizin auf: Politik, die irgendwie mit 
Gesundheit und Krankheit zu tun hat, ist für die Medizin potenziell interessant, und das betrifft eigentlich «all policies» [1].

Dieser ganzheitliche Medizinbegriff findet sein Gegenstück in den Artikeln zur Ausbildung im gegenwärtig gültigen Bundesgesetz über die universitären Medizinalberufe. Dort heisst es nämlich im 6. und 7. Artikel, dass Medizinstudierende verschiedenartige Beziehungen zwischen dem Gesundheitswesen und anderen gesellschaftlichen Zusammenhängen verstehen, dass sie «die Grenzen der medizinischen Tätigkeit» erkennen und die «ethische Dimension [...] und ihre Verantwortung gegenüber Individuum, Gesellschaft und Umwelt wahrnehmen» sollen [2]. Natürlich sind die Medizinkonzepte bei Gilli und im Bundesgesetz historisch spezifische Perspektiven, weil sich Ansichten über Einflussfaktoren und Bildungsziele über die Zeit stark ändern: Vor hundert Jahren hätte beispielsweise niemand von krankmachenden Faktoren wie "Lärm» oder «Stress» (Gillis Beispiele) gesprochen, und es hätte wohl auch niemand die "ethische Dimension" der medizinischen Tätigkeit in den Vordergrund gerückt, weil es Medizinethik als angewandte Ethik in unserem heutigen Sinne nicht gab. Allerdings wäre auch vor hundert Jahren die Ganzheitlichkeit der Medizin zur Diskussion gestanden, die mittels einer bestimmten Disziplin eingeholt werden sollte: mittels der Medizingeschichte.

\section{Der ärztliche Humanismus}

Gemäss Henry Sigerist, dem zweiten Privatdozenten für Medizingeschichte der Universität Zürich, brauchen Studierende der Medizin Kenntnisse der Medizingeschichte. Diese verwandelt nämlich blosse Fachidiotinnen und -idioten in Menschen "von umfassender Geistes- und Seelenbildung». Die Medizingeschichte sei demnach ein «unerlässliches Studien-

\section{Bereits im 19. Jahrhundert war das Fach Medi-} zingeschichte Bestandteil des Curriculums von Medizinstudierenden.

und Wissensgebiet», das den Weg bereitet "zu einem neuen, umfassenden Humanismus, in dem der Arzt die ihm zukommende Stelle des wahren Vollmenschen einnehmen würde» [3]. Der Ruf nach einem ärztlichen Humanismus in der medizinischen Ausbildung und darüber hinaus stammt aus Sigerists Antrittsvorlesung im November 1921 [4]. Sigerists Forderung ertönte nicht im luftleeren Raum. Er bereitete nämlich gerade gemeinsam mit anderen die Grün- dung einer neuen Gesellschaft im Dezember 1921 vor: die Schweizerische Gesellschaft für Geschichte der Medizin und der Naturwissenschaften. Medizingeschichte war aber schon vorher nie nur mit «spielerischem Raritätenkram» beschäftigt gewesen, wie der deutsche Medizinhistoriker Karl Sudhoff seine Disziplin 1913 verteidigt hatte [5]. Vielmehr hatte sie als angewandte Disziplin immer ganz bestimmte Funktionen im Rahmen des ärztlichen Selbstverständnisses und der medizinischen Praxis übernommen.

Die Medizingeschichte sollte Resonanz- und Diskussionsraum für ärztliche Anliegen sein und gewissermassen fachintern wirken.

Bereits im 19. Jahrhundert war das Fach Medizingeschichte Bestandteil des Curriculums von Medizinstudierenden gewesen, doch in den ersten Jahrzehnten des 20. Jahrhunderts erlebte die Disziplin einen regelrechten Höhepunkt mit einer Welle von neuen nationalen und internationalen Gesellschaften für die Medizingeschichte auf der ganzen Welt. Dazu kamen zahlreiche neue Bibliotheken und Sammlungen sowie die Errichtung von Dozierendenstellen. Grund für diesen Institutionalisierungsschub war zunächst die Befürchtung, dass sich Ärztinnen und Ärzte angesichts der sogenannten Verwissenschaftlichung der Medizin im Spezialistentum verlören. 1921 war daher die Stossrichtung der zu gründenden Gesellschaft klar: Die Gesellschaft sollte daran arbeiten, «eine Vertiefung des naturwissenschaftlichen Denkens zu erreichen und den Kontakt zwischen Naturwissenschaften und Geisteswissenschaften inniger zu gestalten» [6].

Sigerist sprach von dieser Brückenfunktion als notwendigem ärztlichem "Humanismus», der über die Medizingeschichte zu erreichen war. Wie die Statuten der SGGMN verdeutlichten: Es ging um das «naturwissenschaftliche» Denken", das es zu erweitern galt. Aus diesem Grund trugen einige der neuen Gesellschaften sowohl die Medizin als auch die Naturwissenschaften in ihrem Namen, neben der SGGMN beispielsweise auch die ehrwürdige Deutsche Gesellschaft für Geschichte der Medizin und der Naturwissenschaften, die 1901 ins Leben gerufen wurde. Angesprochen wurde explizit die Geschichte der Medizin zwischen Kunst, Gesellschafts- und Naturwissenschaft. Natürlich war die SGGMN nicht primär eine universitäre Einrichtung, die auch nicht direkt eine Bildungsfunktion übernehmen sollte. Gleichwohl kann die innergesellschaftliche Debatte über die Rolle der Medizingeschichte kaum von ähnlich gelagerten Diskussionen im Medizincurriculum getrennt 
werden, zumal es immer grosse Überschneidungen zwischen SGGMN-Mitgliedern und den Dozierenden in Medizingeschichte an schweizerischen Bildungsinstitutionen gab [7].

\section{Medizingeschichte als Resonanzraum}

Aus heutiger Sicht wirken einige der zentralen Methoden und Inhalte der damaligen Medizingeschichte ziemlich antiquiert: Übersetzungen und Textausgaben antiker Texte, Bibliographien, und vor allem Biographien grosser Ärzte der Vergangenheit. Es darf aber nicht vergessen werden, dass gerade diese Art von Medizingeschichte den damaligen Bedürfnissen der ärztlichen Zunft entsprach. Dazu gehörte auch das Bedürfnis, mit Medizingeschichte der Fragmentierung und Spezialisierung innerhalb der Medizin entgegenzuwirken und ein Narrativ linearer Entwicklung zu bieten [8]. Sigerist, der zwischenzeitlich von Zürich an die Universität Leipzig gewechselt hatte, sagte von der Geschichte der Medizin in seiner Leipziger Antrittsvorlesung, dass sie nicht ein «Spezialgebiet [...] sondern eine Zusammenfassung» sei, die zur Distanzierung und zum Verständnis der Medizin beitragen sollte [9]. Diese Bemühungen waren kein Selbstzweck, sondern entsprachen bestimmten Vorstellungen von einer guten ärztlichen Persönlichkeit und Tätigkeit. Ärztebiographien stellten natürlich erstklassige Beispiele für dieses Bestreben dar. Auffällig ist jedenfalls, dass in der ärztlichen Ausbildung in «medizinischem Humanismus» des frühen 20. Jahrhunderts die Person der Ärztin oder des Arztes im Zentrum stand. Die Medizingeschichte sollte Resonanz- und Diskussionsraum für ärztliche Anliegen sein und gewissermassen fachintern wirken.

\section{Lange lag die Disziplin in den Händen von} Ärztinnen und Ärzten, die zum Teil geisteswissenschaftliche Ausbildungen hatten.

Gerade weil die Medizingeschichte als Resonanz- und Diskussionsraum für ärztliche und damit auch gesellschaftliche Anliegen fungierte, wurde eine zentrale Frage immer wieder neu gestellt und beantwortet: Wozu eigentlich Medizingeschichte? Eng damit verbunden waren die beiden Fragen nach dem «Wer?» und «Für wen?». Wer schreibt eigentlich die Geschichte der Medizin, und wer rezipiert Medizingeschichte? Lange Zeit lag die Disziplin in den Händen von Ärztinnen und Ärzten, die in manchen Fällen über eine geisteswissenschaftliche Ausbildung verfügten. Die erwähnte Professionalisierung und die Institutionalisierung der
Medizingeschichte innerhalb der Geschichtswissenschaft legt die Ambivalenzen dieser Neuverhandlung offen. Im mittleren Drittel des 20. Jahrhunderts wurden nämlich aufregende neue Ansätze formuliert, wie medizinhistorische Fragen aussehen konnten. Beispielsweise entstand in den 1930er Jahren eine Forschungsgruppe rund um den mittlerweile nach Baltimore emigrierten Henry Sigerist, die Medizingeschichte über das ärztliche Selbstverständnis hinaus als breitere Sozialgeschichte verstand und sich wie im Falle Sigerists auch vor medizinhistorischen Interven-

\section{Mit der Zeit nahm die Schwierigkeit zu,} Forschungsinhalte zurück in die Medizin zu übersetzen.

tionen in die Politik nicht scheute. Nach dem Krieg nahmen viele Medizinhistorikerinnen und -historiker neue methodische und theoretische Anregungen aus den angrenzenden kulturwissenschaftlichen und historischen Disziplinen auf. Zwar blieben medizinhistorische Lehrstühle zumindest im deutschsprachigen Raum in den medizinischen Fakultäten verankert. Die Neuverhandlung und Diversifizierung der medizinhistorischen Disziplin jedoch, im Gespann mit der fortschreitenden Technisierung in der medizinischen Ausbildung, stellte auch die einstige Position von Medizingeschichte in der ärztlichen Wahrnehmung zur Debatte.

\section{Ambivalenz der Medizingeschichte}

Im Rahmen der Geschichte der SGGMN wird diese Ambivalenz auf verschiedene Weisen deutlich. Beispielsweise fanden ab dem Jahr 1950 in Pura für einige Zeit jährliche medizinhistorische Treffen statt. Organisiert wurden diese Treffen von Henry Sigerist, der nach seiner Rückkehr aus den USA im Kanton Tessin seinen Lebensabend verbrachte. Sigerist war offenbar über das SGGMN-Treffen im Jahr 1949 derart unglücklich gewesen, dass er ab dem kommenden Jahr «ernsthafte gleichgesinnte» zu einer Art Parallelveranstaltung einlud [10]. Diese «Pura-Tagungen» zeugten von der Spannung, die sich zwischen der professionalisierten Zunft der Medizinhistorikerinnen und -historiker und den «medizinhistorischen Sonntagsfahrern» ergab [11]. Einige Namen der SGGMN-Präsidenten/Präsidentinnen der folgenden Jahre (z.B. Erwin Heinz Ackerknecht oder Esther Fischer-Homberger) trugen dieser Spaltung Rechnung und werden anders als andere zeitgenössische Medizinhistorikerinnen und -historiker weiterhin gelesen. Die Konsequenz: Mit dem steigenden Interesse innerhalb der professionalisierten 


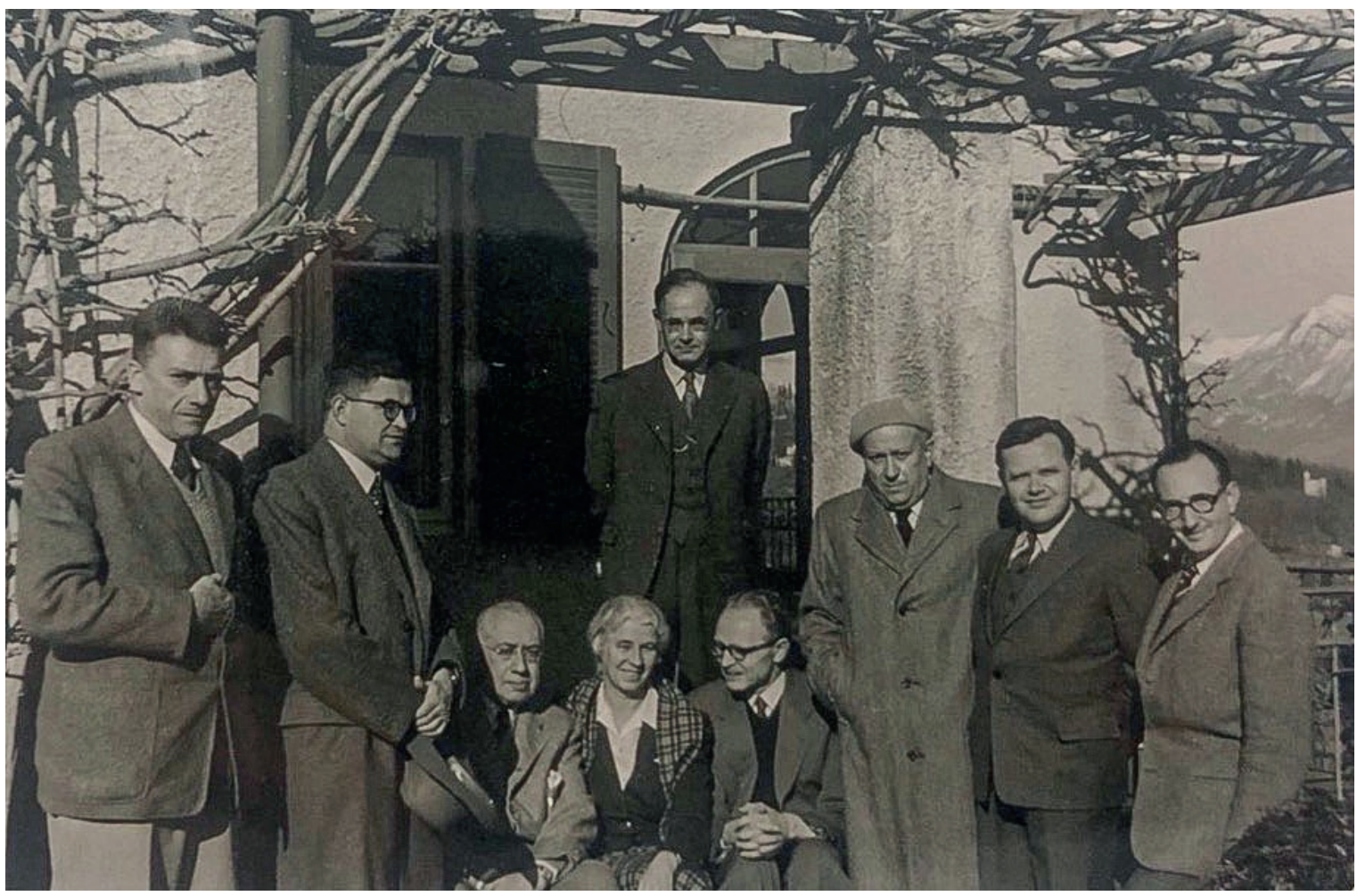

Henry E. Sigerist (dritte Person von rechts, mit Mütze) an der Pura-Tagung umringt von der medizinhistorischen Zunft, 1951.

Geschichtsschreibung nahm allerdings auch die Schwierigkeit zu, die Forschungsinhalte zurück in die Medizin zu übersetzen.

Ab den 1960er Jahren begann sich mit den «medical humanities» in den USA eine neue, zunächst soziologisch orientierte Disziplin zu bilden, die sich durch zwei Dinge auszeichnete: Es ging erstens um eine Kritik der medizinischen Bildung («Entpersonalisierung», Aufstieg der Molekularbiologie, «mechanistische Medizin»), und es handelte sich zweitens um eine Initiative aus der Ärzteschaft [12]. Dazu gesellte sich etwas später die Disziplin der Bioethik, die von Medizinhistorikerinnen und -historikern als ein de-

\section{Das Wichtigste in Kürze}

- In den ersten Jahrzehnten des 20. Jahrhunderts erlebte die Medizingeschichte einen Höhepunkt mit einer Welle von neuen nationalen und internationalen Gesellschaften auf der ganzen Welt.

- Die Medizingeschichte sollte ursprünglich Resonanz- und Diskussionsraum für ärztliche Anliegen sein und gewissermassen fachintern wirken.

- Heute ist die Medizingeschichte eine historische Disziplin. Sie sucht eine enge Anbindung an medizinische Fakultäten, praktizierende Ärztinnen und Ärzte und Akteurinnen und Akteure im Gesundheitssystem, steht aber auch im Austausch mit Fachhistorikerinnen und -historikern. kontexualisiertes und seelenloses Beratungsunternehmen karikiert wurde [13]. Des ungeachtet erfüllte die Bioethik gewisse Bedürfnisse der Ärzteschaft, und auch die um weitere (auch ethische) Herangehensweisen erweiterten «medical humanities» erfreuten sich grosser Nachfrage. Das Aufkommen der sogenannten «medical humanities» und von Disziplinen wie der Bioethik oder der Medizinsoziologie in der zweiten Hälfte des 20. Jahrhunderts sowie die Professionalisierung und Institutionalisierung der Medizingeschichte innerhalb der Geschichtswissenschaften müssen also neben anderen Faktoren auch wesentlich als Neuverhandlung der Bedürfnisse von Ärztin-

\section{L'essentiel en bref}

- L'histoire de la médecine a atteint son apogée durant les premières décennies du $X X^{\mathrm{e}}$ siècle avec la création de nou velles sociétés nationales et internationales dans le monde entier.

- A l'origine, I'histoire de la médecine se voulait un espace de résonance et de discussion des préoccupations médicales et devait davantage servir à l'interne.

- L'histoire de la médecine est aujourd'hui une discipline his torique. Elle cherche à créer des liens étroits avec les facul tés de médecine, le corps médical en exercice et les acteurs du système de santé, sans oublier l'échange avec des historiennes et historiens. 
"Covid, History, and the Medical

Humanities»

Annual Meeting of the Swiss Society of the History of Medicine and Sciences (SGGMN)

26. November 2021

Universität Zürich, Rämistrasse 69, 8001 Zürich

SOC-1-106

9.00-17.30 Uhr

Interessierte sind herzlich willkommen.

Für genauere Informationen: leander.diener[at]uzh.ch

nen und Ärzten verstanden werden. Es ging und geht dabei immer um eine Aushandlung der didaktischen Oberhoheit über den «ärztlichen Humanismus». Heutzutage macht dies genau die Spannung der Medizingeschichte aus. Einerseits sucht die Disziplin eine enge Anbindung an medizinische Fakultäten, an praktizierende Ärztinnen und Ärzte und an Akteurinnen und Akteure im Gesundheitssystem, andererseits ist sie eine historische Disziplin im Austausch mit Fachhistorikerinnen und -historikern, die der Verwertung von Geschichte ausserhalb der Akademie jedoch oft etwas misstrauisch begegnen.

Am 4. Dezember 2021 feiert nun die Schweizerische Gesellschaft für Geschichte der Medizin und der Naturwissenschaften (SGGMN) ihren einhundertsten Geburtstag. Aus diesem Anlass steht die diesjährige Jahresversammlung unter besonderem Zeichen. Die Tagung, die am 26. November 2021 in Zürich stattfinden wird, widmet sich einem hochaktuellen Thema: "Covid, History, and the Medical Humanities». Insbesondere wird die Frage danach gestellt, welche besonderen Herausforderungen und allenfalls neuen Perspektiven sich aus den Erfahrungen der CovidPandemie im Nachdenken über Medizin ergeben. Damit führt die Jahresversammlung in bestem Sinne vor Augen, dass die SGGMN und insbesondere die Medizingeschichte (stellvertretend für die «medical humanities») auch nach hundert Jahren nicht ein- fach mit Vergangenem betraut ist, sondern als Geschichte der Gegenwart fungiert und damit «Einsicht in die conditio humana, ins Leiden, in die Persönlichkeit, in unsere gegenseitige Verantwortung» zu vermitteln versucht, ganz im Sinne der Definition von "medical humanities» durch die League of European Research Universities (LERU) aus dem Jahr 2012 [14].

\section{Bildnachweis}

«Pura-Tagungen», PN 146.4: 374-380. Archiv für Medizingeschichte, Universität Zürich.

Einstiegsbild:

Hush Naidoo Jade Photography / Unsplash

\section{Literatur}

1 Gilli Y. 'Health in all policies', aber nicht 'All policies in health'. Schweiz Ärzteztg. 2021;102(26):868.

2 MedBG 811.11 3. Kapitel: Universitäre Ausbildung. Art. 7 Soziale Kompetenz und Persönlichkeitsentwicklung.

3 Sigerist H. Aufgaben und Ziele der Medizingeschichte. Antrittsvorlesung von Dr. med. Sigerist. In: Neue Zürcher Zeitung, Donnerstag, 16. Februar 1922 (2).

4 Der erste Privatdozent war Gustav Adolf Wehrli, der sich ein Jahr vor Sigerist habilitierte. Fischer H. 50 Jahre Schweizerische Gesellschaft für Geschichte der Medizin und der Naturwissenschaften. Gesnerus. 1971;28:75.

5 Sudhoff K. Was ist Geschichte der Medizin? (1913). In: Ders. Skizzen. Leipzig: F.C.W. Vogel; 1921 (18).

6 Statuten der Schweizerischen Gesellschaft für Geschichte der Medizin und der Naturwissenschaften, 1922.

7 Bickel H, M.H. Editorial: 75 Jahre Schweizerische Gesellschaft für Geschichte der Medizin und der Naturwissenschaften. Gesnerus. 1996;53:169-10.

8 Barras V, Steinke H. Editorial. Gesnerus. Swiss Journal for the History of Medicine and Science, 1943-2020. In: Gesnerus. 2020;77:168

9 Sigerist S. Die historische Betrachtung der Medizin. Archiv für Geschichte der Medizin. 1926;18:19.

10 «Notizen zu den Henry Sigerist-Tagungen» (1). PN 148.1: 859. Medizinhistorisches Archiv, UZH.

11 Erwin Heinz Ackerknecht verwendete Henry Sigerist gegenüber einmal die Bezeichnung «medico-historical Sunday drivers» für sich medizinhistorisch betätigende KlinikerInnen. Rosenberg CE. Erwin H. Ackerknecht, Social Medicine, and the History of Medicine. In: Bulletin for the History of Medicine. 2007;81:512.

12 Fox DM. Who we are: The political origins of the medical humanities. Theoretical Medicine. 1985;6:327-42.

13 Wilson D. What can history do for bioethics? bioethics. 2013;27:21523.

14 League of European Research Universities (LERU). Social Sciences and Humanities: Essential Fields for European Research and in Horizon 2020. Advice Paper No. 11 (Juni 2012). 